\title{
Barriers to nurse-patient communication in Saudi Arabia: an integrative review
}

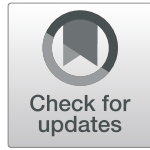

Mukhlid Alshammari ${ }^{*}$ D, Jed Duff and Michelle Guilhermino

\begin{abstract}
Background: Effective nurse-patient communication is important in improving quality of health care. However, there are several barriers to nurse-patient communication in Saudi Arabia. This is attributed to the increasing number of non-Saudi expatriate nurses providing health care to patients. In particular, there are differences in culture, religion and language among non-Saudi nurses and patients. This integrative review aims to identify and synthesize quantitative and qualitative evidence on the current practice in nurse-patient communication in Saudi Arabia and its effect on service users' quality of care, safety and satisfaction.

Methods: An integrative review based on Whittemore and Knafl's approach (Whittemore and Knafl, J Adv Nurs 52: $546-553,2005)$ was used to conduct the review. Peer-reviewed articles containing any of a series of specific key terms were identified from sources such as CINAHL, EMBASE, Medline, PubMed and PsychINFO. The review included studies that focused on nurse-patient communication issues, communication barriers, and cultural and language issues. The search was limited to papers about the Saudi Arabian health system published in English and Arabic languages between 2000 and 2018. A data extraction form was developed to extract information from included articles.

Results: Twenty papers were included in the review (Table 1). Ten papers employed quantitative methods, eight papers used qualitative methods and two used mixed methods. The review revealed two major themes: 'current communication practices' and 'the effect of communication on patients'. Some of the communication practices rely on non-verbal methods due to a lack of a common language, which often results in the meaning of the communication being misinterpreted. Many non-Saudi nurses have limited knowledge about Saudi culture and experience difficulty in understanding, and in some cases respecting, the cultural and religious practices of patients. Further, limited nurse-patient communication impacts negatively on the nurse-patient relationship, which can affect patient safety and lead to poor patient satisfaction.

Conclusions: Current nurse-patient communication practices do not meet the needs of Saudi patients due to cultural, religious and language differences between nurses and patients. The barriers to effective nurse-patient communication adversely effects patient safety and patient satisfaction. Further research from the perspective of the patient and family is needed.
\end{abstract}

Keywords: Saudi Arabia, Nurse-patient communication, Communication barriers, Quality of nursing care, Patient satisfaction

\footnotetext{
* Correspondence: mukhlid.alshammari@uon.edu.au;

mukhlid1983@gmail.com

School of Nursing and Midwifery, University of Newcastle, Callaghan,

Australia
}

(c) The Author(s). 2019 Open Access This article is distributed under the terms of the Creative Commons Attribution 4.0 International License (http://creativecommons.org/licenses/by/4.0/), which permits unrestricted use, distribution, and reproduction in any medium, provided you give appropriate credit to the original author(s) and the source, provide a link to the Creative Commons license, and indicate if changes were made. The Creative Commons Public Domain Dedication waiver (http://creativecommons.org/publicdomain/zero/1.0/) applies to the data made available in this article, unless otherwise stated. 


\section{Background}

The concept of communication is a complex process of exchanging information, thoughts and feelings between individuals using a common system of signs, symbols or behaviors. This process consists of several components, including sender, receiver, context, medium, message and feedback. For communication to occur, a message (information, thoughts and feelings) is transmitted by the sender (also called the encoder) through a suitable medium in a given context to a receiver (also called the decoder), who then provides feedback [1].

In the health-care setting, several theoretical and conceptual approaches have been employed to improve health outcomes, including patient-centered communication [2-6]. Patient-centered communication has been identified as an essential component in delivering quality health services [4]. High-quality patient-centered communication has been shown to help patients strengthen their relationship with nurses, handle their emotions, understand important information regarding their illness, deal with uncertainty, and participate more fully in decisions regarding their health $[2,4]$.

Nurse-patient communication plays an important role in improving not only patient's relationship with the nurse, but also the patient's own perception of the treatment process and outcome. Moreover, having effective communication skills is essential for health-care providers' practice and their ability to understand the clinical symptoms and psychological and emotional needs of their patients. Patient-centered communication enables the building of therapeutic relationships, which helps health-care providers apply intelligent, sensitive and collaborative approaches to communicate with patients about their services $[2,5,7-10]$.

Despite the potentially significant benefits of patientcentered communication, there have been communication barriers identified across a number of different practice settings worldwide [11-14]. For instance, limited knowledge and understanding of the culture and language of a health system on the part of a patient has been shown to limit the communication process between patient and clinician [13-16]. These barriers are influenced by several factors including cultural and language diversity [12]. These communication barriers can affect health outcomes, quality of health care, patient safety and patient satisfaction.

Nurse-patient communication is a challenge in the Saudi Arabia health system because many of the nurses are expatriates and don't speak Arabic. This issue is not unique to Saudi Arabia, due to increasing levels of immigration into developed countries such as United States of America and Australia, there is increasing cultural and linguistic diversity between nurses and their patients $[12,14]$. In fact, a recent systematic review [13] suggested that such communication barriers are common to many countries and they adversely affect the overall quality of health services.

There has been growing interest in research on nursepatient communication in Saudi Arabia, including quantitative and qualitative studies [17-21], but despite the growing evidence base no study has focused on the communication experiences of patients. Furthermore, no study has examined if the patient's communication experience impacts satisfaction with their nursing care.

\section{Aim}

This integrative review aims to identify and synthesize quantitative and qualitative evidence on the communication practices among nurses and patients in Saudi Arabia and their effect on patient satisfaction, quality of care and safety.

\section{Methods}

An integrative review was chosen to merge diverse methods, and synthesize findings from both qualitative and quantitative studies [22]. The integrative review used Knafl and Whittemore's methodology [23]. This approach involved a five-stage process: (1) identify the purpose of the review, (2) search the relevant literature, (3) evaluate and extract data, (4) analyze or synthesize the data, and (5) present findings [23]. The quality of the included papers in this review were evaluated using the Mixed Methods Appraisal Tool (MMAT) [24].

\section{Inclusion criteria and search strategy}

The review included papers written in Arabic and English and focused on Saudi Arabian healthcare. Studies were included if they focused on nurse-patient communication, including communication barriers such as language and cultural issues and their effects on patients.

CINAHL, EMBASE, PubMed, Medline and PsychINFO databases as well as Google Scholar were searched for articles published between 2000 and 2018 . These databases and the time limit were chosen to ensure a comprehensive search and a sufficient breadth and depth in the retrieved literature. A two-stage search approach was utilized to facilitate the search process. An initial search was conducted in Medline and EMBASE. Subsequently, the identified key words and search items were modified and used to search across CINAHL, PUBMED and PsychINFO. The search items that facilitated the search process were barrier* or facilitat* or limit* or challeng* or difficult* or obstacle* or problem or issue AND communicat* or language or cultur* AND nurs* AND Saudi* AND quality or satisf* or effectiv* or impact. 


\section{Data extraction and synthesis}

The integrative review contained several stages in the selection of papers. The Preferred Reporting Items for Systematic Reviews and Meta-Analyses (PRISMA) flow chart (Fig. 1) represents the process. First, the titles and abstracts of all the papers identified from the scientific databases were screened against the inclusion criteria. Second, the abstracts of all the included titles were reviewed to identify those that required full text review. The final stage of the selection process involved screening the full text articles to agree on those to include in the final synthesis. Two reviewers independently managed the selection process.

A pre-defined data extraction form was developed to guide the process of data extraction. The tool was developed and structured according to the systematic review reporting guidelines [25]. The data extraction form was structured into sub-sections, which included description of study (author, year of publication and title of paper), methods (study design, methods, sampling and sample size) and findings.

\section{Critical appraisal}

The quality of the papers was assessed using the Mixed Methods Appraisal Tool (MMAT). This tool has been validated and widely used to asses quality of papers with different methods [24]. MMAT has three categories of quality score, including low (a score below 25\%), medium (a score of 50\%) and high (a score of 75\% and above). All of the included studies were checked on the basis of data relevance as well as methodological rigor.

\section{Results}

\section{Study characteristics}

A total of 150 records were reviewed from CINAHL, EMBASE, PubMed, Medline, PsychINFO and Google Scholar, of which 26 duplicates were deleted. From this, 124 titles and abstracts were assessed against the inclusion criteria, with 79 excluded. A full text review of the remaining 45 documents was undertaken. Twenty five manuscripts were later excluded, 10 because of the population, seven were excluded because it did not focus on nurse-patient communication issues and its effect on health outcomes; and eight were excluded because the study setting did not include Saudi Arabia healthcare context. Overall, data was extracted from 20 full text articles and included in the final synthesis (Fig. 1). Of the 20 papers included, eight used qualitative methods, 10 used quantitative methods and two used mixed methods (Table 1). Further, 15 of the included studies targeted

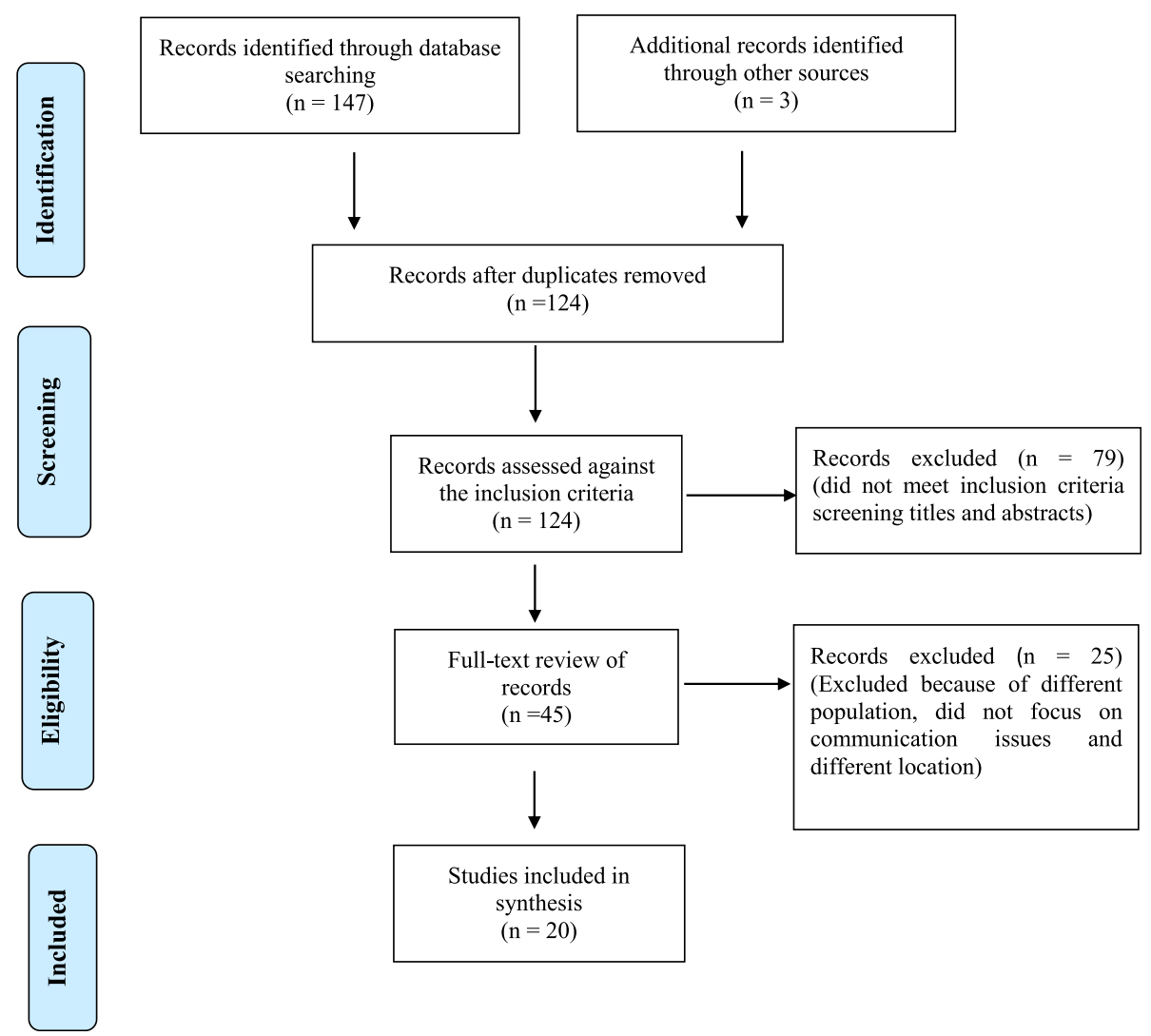

Fig. 1 PRISMA chart of article selection 


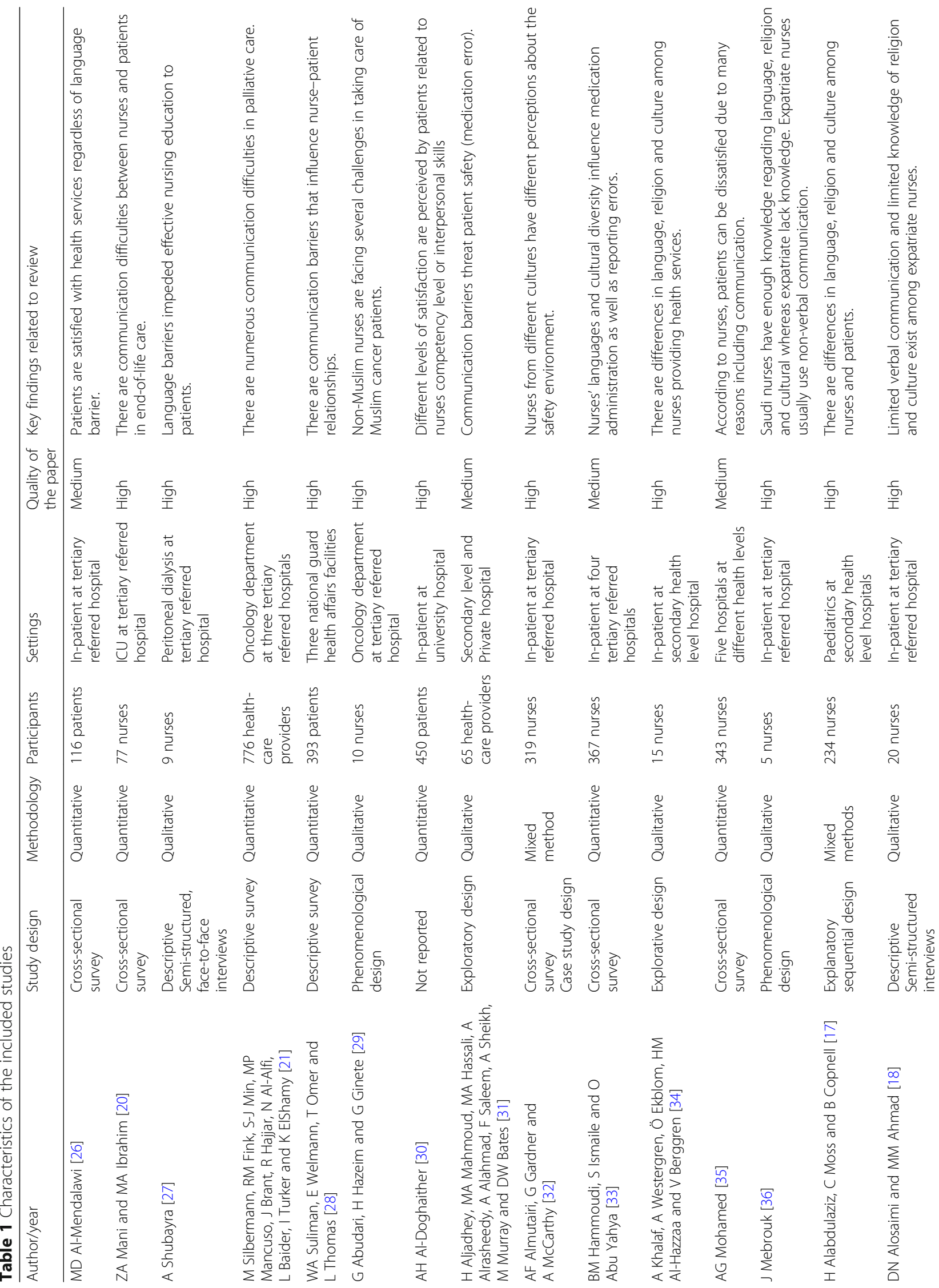




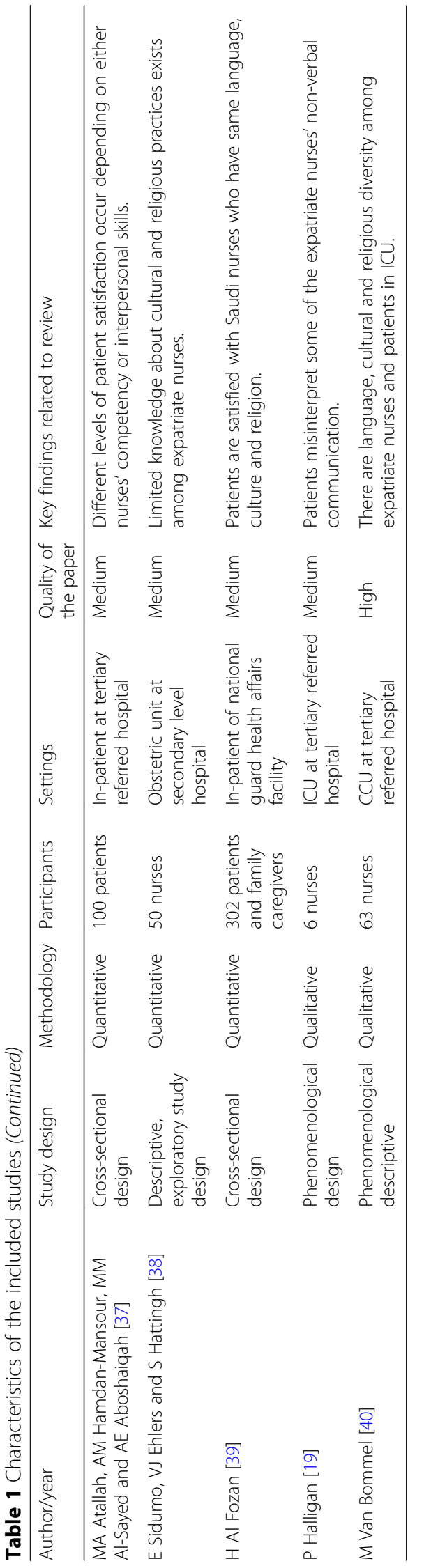


nurses' perspectives of the communication and five focused on patients' perspectives of communication. A quality assessment of the included papers found that most (12 out of 20) were asssessed as high quality, while the remaining papers (eight out of 20) were assessed as medium quality.

\section{Identified themes}

The major themes were grouped into two themes: 'current communication practices' and 'the effect of communication on patients' (Table 2). The sub-themes identified from the 'current communication practices' theme were language, religion and cultural diversity; communication practices; and communication barriers. The sub-themes identified from the 'effect of communication on patients' theme were quality of care and patient satisfaction.

\section{Current communication practices}

In Saudi Arabia, the nursing workforce across almost all health facilities is dominated by non-Saudi nurses, primarily from the Philippines and India, supplemented by nurses from the USA, UK, Australia and various European countries [40]. The increasing number of nonSaudi or expatriate nurses has created several challenges in the delivery of health care. In particular, the challenges are ascribed to several factors, mostly linked to cultural, language and religion differences. The current communication practices present a barrier to patientcentered interaction between nurses and patients [19, 29]. These challenges are described below.

\section{Language, religion and cultural diversity}

Seven of the included papers explained that there are differences in language, religion and culture among nurses providing health services to patients in Saudi Arabia [17-20, 34, 38, 40] with the language, religion and culture of non-Saudi nurses differing from their Saudi patients. Unlike the expatriate nurses, almost all patients in Saudi Arabia speak Arabic and share the same cultural values, norms and religion [40].

Khalaf, A et al. (2014) stated that the religious-cultural norms and values of Saudi patients appear as entirely new to many non-Saudi nurses [34]. Some cultural or religious practices, such as gender segregation or females covering their hair or faces, do not appear to be rational to many nurses. In addition, some religious-cultural practices, which lead female patients to have a preference for female practitioners when seeking health care, are sometimes seen as irrational by non-Saudi nurses.

Consequently, multiple studies have found that nonSaudi nurses experience difficulty in understanding, and in some cases respecting, the cultural and religious practices of patients [20,38]. This was attributed to the fact that most of these expatriate nurses have limited knowledge about the practices of their patients. In particular, the nurses have limited knowledge about the cultural and religious practices of patients [18, 38]. For instance, a previous study showed that more than half of all expatriate nurses lack knowledge of Saudi culture [38]. Practically, this makes it difficult for the nurses to understand the communication dynamics of patients in the process of seeking health care.

The differences in language, culture and religion are significant factors that can directly influence the communication experience of the patient. These factors cannot be overlooked in the delivery of health care $[17,19$, 38]. For example, cultural and traditional practices including the use of herbal medicine, preference for breastfeeding and practice of burying the placenta are believed to improve health and prevent illness of both baby and mother. Along with strong family bonds, these factors have historically played a significant role in delivery of Saudi Arabian health care. In addition, religious beliefs and practices such as fasting and praying are perceived as relevant factors in the recovery of patients [36, 40]; however, some non-Saudi nurses have demonstrated difficulty in understanding these religious-cultural practices that contribute significantly in the delivery of health care.

Nor is this a new phenomenon. Differences in language between nurses and patients have historically created problems in the health systems of Saudi Arabia, particularly in some routines of nurses. Two studies identified that language diversity causes significant challenges in caring for patients with life-threatening conditions; in particular, when discussing patient wishes in terms of their care and during sessions of health education [20, 29]. Other studies identified that nurses experienced difficulty in understanding patients, particularly

Table 2 Emerging themes and sub-themes

\begin{tabular}{lll}
\hline Theme & Sub-theme & Number of papers \\
\hline Current communication practices & Language, religion and cultural diversity & 7 \\
& Communication practices & 4 \\
Effect of communication on patients & Communication barriers & 6 \\
& Quality of care and patient safety & 5 \\
\hline
\end{tabular}


when seeking information during health-care delivery, such as taking the history of the patient $[18,19]$. Several studies have suggested that the phenomenon not only poses significant challenge to the nurses but also to the patients and their families [17, 20, 34]. In particular, some nurses avoid conversations with patients or their families due to language differences.

\section{Communication barriers}

Six studies have shown that there are numerous interpersonal therapeutic communication barriers existing between nurses and patients as well as family caregivers [17-21, 27]. These barriers occur at different levels, including nurses to patients as well as at the interprofessional level $[21,34]$. The inter-professional communication barriers are the barriers occurring at the health provider level, mostly between nurses from Saudi Arabia and non-Saudi expatriate nurses. The communication barriers occurring at different levels of the health system are ascribed to several factors, again mostly linked the differences in language, culture and religion $[17,18,20,26,29,34]$.

Consequently, the limited Arabic language, culture and religion knowledge have negatively impacted on the delivery of health care, particularly by limiting the communication processes between non-Saudi expatriate nurses and patients $[17,18,20]$. In particular, the language difficulties limit nurses' ability to effectively communicate with patients $[17,18,20]$. Similarly, the communication barriers occurring at inter-professional level affect health services planning [31].

As well as the in-service training programs noted above, the health authorities have employed several strategies to overcome these challenges, particularly at the health facility level. As described by Almutairi, AF et al. (2013), these strategies include the use of interpreters or family member to translate conversations [32]. Although these strategies can be useful, they are not always as effective as needed. For example, both translators and family members have been shown to deliver an incomplete or unclear rendering of the conversation to the patient, which could adversely influence health-care practice [32].

It has been argued that the provision of interpreters in all hospitals in Saudi Arabia would be a good step towards improving communication [32]. However, many of these translators would need training in personal and professional interpretive skills, particularly in the area of medication administration [41]. Although most current interpreters are competent in the English language, their skills are limited to the understanding of medical terms and jargon.

\section{Communication practices}

Four studies identified communication practices as a key theme. They identified several communication practices currently employed by nurses in health facilities across Saudi Arabia that are perceived to be effective in interpersonal therapeutic communication [18, 19, 29, 36]. Existing evidence suggest that most nurses employ nonverbal communication practices in their communication with patients. Predominantly, this takes the form of gestures and signs, and sometimes therapeutic touch as well as smiling [29, 36]. In some instances, these non-verbal communication practices help patients understand the process of health-care delivery. In particular, recent evidence suggests that the non-verbal communication appears to reassure patients and their families about the medication processes, which provides them with a degree of relief [29].

However, despite the increasing use of non-verbal communication practices, two studies have highlighted that such communication is frequently misinterpreted by patients $[19,36]$. Two examples are the clicking of fingers to attract the patient's attention, and the use of direct eye contact with patients. To Saudi patients, the clicking of fingers to attract attention can be understood as offensive [19], while direct eye contact by female nurses to male patients could be understood as a rude behavior [36].

In addition to the non-verbal communication, some nurses communicate verbally to facilitate patientcentered interaction [18]. The verbal interactions are usually limited and delivered through few Arabic/Islamic terms. A recent study suggested that words such as "Bismillah" or "Alhamdillah" - which translate as "in the name of Allah" and mean to start with the blessing of God - are mostly used prior, during and after medication processes, largely to make the patient feel more comfortable [18].

The Ministry of Health in Saudi Arabia has responded to these issues by instituting cultural training and orientation programs for nurses. This training is delivered as an in-service program with the primary aim of exposing nurses to religious-cultural practices in the delivery of health care in Saudi Arabia [40]. However, despite this development, two studies have suggested that these courses appear to have limited impact and lack the ability to meet the needs of such nurses [32, 40]. In particular, this training and orientation program has a short duration and scope, and it has been noted that in order to address such communication gaps, improvements would be required in the content, structure, duration and intensity of the program [32].

\section{Effect of communication on patients Quality of care and patient safety}

Five studies suggested that a comprehensive understanding of the culture, religion and language of a geographical setting play significant role in improving the quality 
of care and safety of the population [27, 28, 31-33]. In particular, nurses who have some knowledge and understanding of Saudi religious-cultural practices are perceived to be more competent in delivering care compared to those with limited competency [31]. For instance, some expatriate nurses continue to struggle with communication and subsequently feel frustrated, particularly in understanding aspects of the patient's culture and religion [19]. One study proposes that a deep understanding of some elements of the religious-cultural attributes - such as Muslim lifestyle, hygienic practices, ways of dressing, and gender segregation by nurses - is necessary to improve the quality of care and safety of patients [40].

Four studies highlighted that communication barriers caused by the differences in religious-cultural practices have implications for the safety of the patients [27, 31-33]. These challenges are experienced in areas such as medication safety and the emotional, psychological, physical and spiritual domains of patients and family members. In some instances, the challenges affect not only patients but also the nurses delivering care to patients.

Three studies suggested that where nurses have religious-cultural practices that differ from those of their patients, safety can be impacted [27, 32]. For example, patients may find it difficult to adhere to the nurse's instructions, resulting in a clear threat to patient safety $[26,31$, 33]. Another threat to patient safety is caused by miscommunication between nurse and patient or between health professionals. Aljadhey, $\mathrm{H}$ et al. (2014) have suggested that language barriers could account for an increase in medication errors [31]. Similarly, Hammoudi, BM et al. (2017) found that some non-Saudi nurses are hesitant to report medication errors or subsequent adverse effects on the patient for fear of disciplinary action [33].

\section{Patient satisfaction}

Five studies highlighted that patients exhibit different levels of perceived satisfaction from different aspects of health care in Saudi Arabia [26, 30, 35, 37, 39]. Approximately $75 \%$ of patients reported being satisfied with the health care they have previously received; however, while these patients are generally satisfied with the technical competence of nurses [30, 37], approximately half of them are dissatisfied with the interpersonal therapeutic communication of most expatriate nurses.

In particular, nurses who share the same language, culture and religion as the patient are perceived to communicate professionally and clearly, to respect culture and religion, and to maintain patient's privacy. This contributes to building a good relationship between nurse and patient, resulting in an improved satisfaction with patient care $[36,37,39]$. Although patients recognize that expatriate nurses are generally technically competent, patients are less satisfied with interpersonal therapeutic communication, as they perceive nurses to be ignorant of their language, culture and religion [37]. This can be perceived as disrespect, which might contribute to reported levels of violence towards nurses. A previous study highlighted that communication barriers was one of the important factors in work-related violence [35].

\section{Discussion}

This review aimed to synthesize evidence on nurse-patient communication practices among nurses and patients in Saudi Arabia and their effect on patients' quality of care, safety and satisfaction. The review included 20 papers in the final synthesis. The review findings suggest that there is a diversity in the language, religion and culture of nurses providing health-care services in Saudi Arabia. In particular, nurses providing health care in Saudi Arabia are largely expatriate and tend to have limited knowledge about Saudi language, religion and culture. Consequently, expatriate nurses rely mostly on non-verbal communication strategies to interact with patients. The review findings suggest that the cultural and language training provided to expatriate nurses is not fit for purpose. Bozionelos [42] qualitative study of 206 expatriate nurses in Saudi Arabia found that nurses are provided with limited face-to-face training due to an overall pressure on nursing services related to the nursing shortage in Saudi Arabia. The limited knowledge about the language, religion and culture of non-Saudi nurses, together with the current communication practices described earlier, have significantly contributed to nurse-patient communication barriers in Saudi Arabia. The review findings suggest that the interpersonal therapeutic communication barriers occur among health care professionals as well as between nurses and patients. In addition, the review findings highlighted that the communication barriers have significant influence on the outcomes of health-care service delivery; in particular, communication barriers have negative effect on the perceived quality of care, patient safety and patient satisfaction.

It is noteworthy that no study has addressed nurse-patient communication experiences from patient perspectives in Saudi Arabia. Given the nurse-patient communication challenges discussed in this paper, future patients with complex needs may face specific challenges in accessing health-care services due to the nature of their conditions, which requires frequent attention from nurses. In particular, patients may require attention in therapeutic communication to make complex and significant medical decisions. In addition, some patients may need more attention from nurses in the process of treatment. Consequently, to improve access to treatment for patients, it is important not only to understand the burden of their conditions but also 
to address the therapeutic communication issues with service providers, particularly nurses.

Based on these review findings and Bozionelos [42] study, some of the recommendations for clinical and policy practices should include the provision of adequate cultural and language training before expatriate nurses leaving their home country; and the implementation of mentorship programs to support and guide expatriate nurses [42]. This can help to improve the communication between nurses and patients in the delivery of health care services. Second, the current in-service training curriculum for nurses in Saudi Arabia should be expanded, and incorporate a component on language, cultural and religious practices. This ought to carry through at least the first year of employment for all nurses, include a formal evaluation component, and be reviewed regularly by the Saudi Ministry of Health for quality and effectiveness.

In addition, the review findings recommend the following in future research. First, because current studies on nurse-patient communication issues largely use the perspective of nurses, with limited studies focusing on patients, particularly those with complex needs, future research should aim at investigating the perspectives of patient and family members on nurse-patient communication issues. Second, researchers and clinicians should aim to use a mixed methods approach to examine the perspectives of both nurses and patients on communication issues. This can help achieve convergence in data analysis. Finally, future research should aim at designing interventional studies to examine the effectiveness of the nurse-patient communication strategies on patient satisfaction and health outcomes.

\section{Strength and limitations}

The strengths of this study include the use of a systematic approach to search data from relevant scientific databases, revealing most available papers on the subject of nurse-patient communication and associated issues such as cultural, religious and language challenges, perceived quality of care, patient safety and patient satisfaction.

Second, the review used a data extraction form to extract all relevant information that met the inclusion criteria. The data extraction form was developed using relevant methodological standards and criteria. Similarly, the review was not limited to any particular sphere of health care, and so could identify all relevant papers, irrespective of any particular health condition being examined.

The study also has several limitations. First, the study was necessarily limited to a selection of specific search items, and so could have missed some relevant papers. Further, the study was limited to papers focusing on
Saudi Arabian health care, and so cannot be generalized to other settings. However, the use of a systematic approach [23] - including data searching, data extraction and collaboration with experts in the field - attempted to reduce the impact of the limitations.

\section{Conclusion}

The study concludes that language, culture and religion differences exist between patients and nurses in Saudi Arabia, primarily due to the preponderance of expatriate nurses in working in the Saudi Arabian health-care system. These differences create barriers to clear and effective communication and produce a negative impact on health outcomes for patients in Saudi Arabia. Moreover, the findings of this review indicate a need to improve communication between patients and health-care providers in order to provide safety and high-quality practice in Saudi Arabia, which will contribute to higher quality of care and patient satisfaction. There should be a focus on research in extensive training programs for nurses.

\section{Abbreviations}

MMAT: Mixed Methods Appraisal Tool; PRISMA: Preferred Reporting Items for Systematic Review and Meta-analysis

\section{Acknowledgements}

The authors want to thank the University of Newcastle Australia. The authors want also to thank Debbie Booth, University of Newcastle librarian for supporting the literature search.

\section{Authors' contributions}

The authors conceptualized the study. MA conducted the data extraction. JD and MG managed the second review of the data extraction. MA, JD and MG worked closely to draft the manuscript. All of them have read and approved the final manuscript.

Funding

No funding was required to complete this study.

Availability of data and materials

All data generated or analyzed during this study are included in this published article (and its supplementary information files).

Ethics approval and consent to participate

Not applicable.

Consent for publication

Not applicable.

Competing interests

The authors declare that they have no competing interests.

Received: 29 April 2019 Accepted: 15 November 2019

Published online: 03 December 2019

\section{References}

1. Lunenburg FC. Communication: the process, barriers, and improving effectiveness. Schooling. 2010;1(1):1-11.

2. McCormack LA, Treiman K, Rupert D, Williams-Piehota P, Nadler E, Arora NK, Lawrence W, Street RL Jr. Measuring patient-centered communication in cancer care: a literature review and the development of a systematic approach. Soc Sci Med. 2011;72(7):1085-95.

3. Treiman K, McCormack L, Olmsted M, Roach N, Reeve BB, Martens CE, Moultrie RR, Sanoff H. Engaging patient advocates and other stakeholders 
to design measures of patient-centered communication in cancer care. Patient Centered Outcomes Res. 2017;10(1):93-103.

4. Treiman K, McCormack L, Wagner L, Roach N, Moultrie R, Sanoff H, Bann C, Street RL Jr, Ashok M, Reeve BB. Factors affecting the communication experiences of newly diagnosed colorectal cancer patients. Patient education and counseling; 2018.

5. Blanch-Hartigan D, Chawla N, Beckjord El, Forsythe LP, de Moor JS, Hesse BW, Arora NK. Cancer survivors' receipt of treatment summaries and implications for patient-centered communication and quality of care. Patient Educ Couns. 2015:98(10):1274-9.

6. Epstein R, Street RL. Patient-centered communication in cancer care: promoting healing and reducing suffering. Bethesda: National Cancer Institute, US Department of Health and Human Services, National Institutes of Health; 2007.

7. Washington GT. The theory of interpersonal relations applied to the preceptor-new graduate relationship. J Nurses Prof Dev. 2013;29(1):24-9.

8. Senn JF. Peplau's theory of interpersonal relations: application in emergency and rural nursing. Nurs Sci Q. 2013;26(1):31-5.

9. Sheldon LK. Communication for nurses: talking with patients. United States: Jones \& Bartlett Learning; 2009.

10. Bello O. Effective communication in nursing practice: a literature review; 2017

11. Fleischer S, Berg A, Zimmermann M, Wüste K, Behrens J. Nurse-patient interaction and communication: a systematic literature review. J Public Health. 2009;17(5):339-53.

12. Keatinge D, Bellchambers H, Bujack E, Cholowski K, Conway J, Neal P. Communication: principal barrier to nurse-consumer partnerships. Int J Nurs Pract. 2002;8(1):16-22.

13. Andriyanto A. Communication barrier between nurse and patient at the hospital: a systematic review. J Health Policy Manag. 2019;4(2):105-10.

14. Cowan DT, Norman I. Cultural competence in nursing: new meanings. J Transcult Nurs. 2006;17(1):82-8.

15. Anderson J, Perry J, Blue C, Browne A, Henderson A, Khan KB, Kirkham SR, Lynam J, Semeniuk P, Smye V. "Rewriting" cultural safety within the postcolonial and postnational feminist project: toward new epistemologies of healing. Adv Nurs Sci. 2003;26(3):196-214

16. Anoosheh M, Zarkhah S, Faghihzadeh S, Vaismoradi M. Nurse-patient communication barriers in Iranian nursing. Int Nurs Rev. 2009:56(2):243-9.

17. Alabdulaziz H, Moss C, Copnell B. Paediatric nurses' perceptions and practices of family-centred care in Saudi hospitals: a mixed methods study. Int J Nurs Stud. 2017;69:66-77.

18. Alosaimi DN, Ahmad MM. The challenges of cultural competency among expatriate nurses working in Kingdom of Saudi Arabia. Res Theory Nurs Pract. 2016;30(4):302-19.

19. Halligan P. Caring for patients of Islamic denomination: critical care nurses experiences in Saudi Arabia. J Clin Nurs. 2006;15(12):1565-73.

20. Mani ZA, Ibrahim MA. Intensive care unit nurses' perceptions of the obstacles to the end of life care in Saudi Arabia. Saudi Med J. 2017; 38(7):715-20.

21. Silbermann M, Fink RM, Min S-J, Mancuso MP, Brant J, Hajjar R, Al-Alfi N, Baider L, Turker I, ElShamy K. Evaluating palliative care needs in middle eastern countries. J Palliat Med. 2015;18(1):18-25.

22. Pearson A, White H, Bath-Hextall F, Apostolo J, Salmond S, Kirkpatrick P. The Joanna Briggs Institute reviewers' manual 2014: Methodology for JBI mixed methods systematic reviews, vol. 1. Adelaide: The Joanna Briggs Institute; 2014. p. 5-34

23. Whittemore R, Knafl K. The integrative review: updated methodology. J Adv Nurs. 2005;52(5):546-53.

24. Pace R, Pluye P, Bartlett G, Macaulay AC, Salsberg J, Jagosh J, Seller R. Testing the reliability and efficiency of the pilot mixed methods appraisal tool (MMAT) for systematic mixed studies review. Int J Nurs Stud. 2012:49(1):47-53.

25. Kitchenham B: Procedures for performing systematic reviews. Keele, Keele University 2004, 33(2004):1-26.

26. Al-Mendalawi MD. The effect of nurse-patient language barrier on patients satisfaction. Saudi Med J. 2011:32(5):539-40.

27. Shubayra A. Continuous ambulatory peritoneal dialysis: Nurses' experiences of teaching patients. Saudi J Kidney Dis Transpl. 2015;26(2):309.

28. Suliman WA, Welmann E, Omer T, Thomas L. Applying Watson's nursing theory to assess patient perceptions of being cared for in a multicultural environment. J Nurs Res. 2009;17(4):293-300.
29. Abudari G, Hazeim H, Ginete G. Caring for terminally ill Muslim patients: lived experiences of non-Muslim nurses. Palliat Support Care. 2016;14(6): 599-611.

30. Al-Doghaither AH. Inpatients satisfaction with nursing services at king khalid university hospital, Riyadh, Saudi Arabia. J Fam Commun Med. 2000;7(3):37-45.

31. Aljadhey H, Mahmoud MA, Hassali MA, Alrasheedy A, Alahmad A, Saleem F, Sheikh A, Murray M, Bates DW. Challenges to and the future of medication safety in Saudi Arabia: a qualitative study. Saudi Pharm J. 2014;22(4):326-32.

32. Almutairi AF, Gardner G, McCarthy A. Perceptions of clinical safety climate of the multicultural nursing workforce in Saudi Arabia: a cross-sectional survey. Collegian. 2013;20(3):187-94.

33. Hammoudi BM, Ismaile S, Abu Yahya O. Factors associated with medication administration errors and why nurses fail to report them. Scand J Caring Sci. 2017;32:1038-46.

34. Khalaf A, Westergren A, Ekblom Ö, Al-Hazzaa HM, Berggren V. Nurses' views and experiences of caring for malnourished patients in surgical settings in Saudi Arabia-a qualitative study. BMC Nurs. 2014;13(1):29.

35. Mohamed AG. Work-related assaults on nursing staff in Riyadh, Saudi Arabia. J Fam Community Med. 2002;9(3):51.

36. Mebrouk J. Perception of nursing care: views of Saudi Arabian female nurses. Contemp Nurse. 2008;28(1-2):149-61.

37. Atallah MA, Hamdan-Mansour AM, Al-Sayed MM, Aboshaigah AE. Patients' satisfaction with the quality of nursing care provided: the Saudi experience. Int J Nurs Pract. 2013;19(6):584-90.

38. Sidumo E, Ehlers VJ, Hattingh S. Cultural knowledge of non-Muslim nurses working in Saudi Arabian obstetric units. Curationis. 2010;33(3):48-55.

39. Al Fozan $\mathrm{H}$. Patients and family caregivers' satisfaction with care delivered by Saudi nurses at National Guard Health Affairs Hospitals in Saudi Arabia. J Nat Sci Res. 2013;3(12):67-74

40. Van Bommel M. Expatriate non-Muslim nurses' experiences of working in a cardiac intensive care unit in Saudi Arabia; 2011.

41. Almutairi AF, Rondney P. Critical cultural competence for culturally diverse workforces: toward equitable and peaceful health care. Adv Nurs Sci. 2013 36(3):200-12.

42. Bozionelos N. Expatriation outside the boundaries of the multinational corporation: a study with expatriate nurses in Saudi Arabia. Hum Res Manag. 2009;48(1):111-34.

\section{Publisher's Note}

Springer Nature remains neutral with regard to jurisdictional claims in published maps and institutional affiliations.

Ready to submit your research? Choose BMC and benefit from:

- fast, convenient online submission

- thorough peer review by experienced researchers in your field

- rapid publication on acceptance

- support for research data, including large and complex data types

- gold Open Access which fosters wider collaboration and increased citations

- maximum visibility for your research: over $100 \mathrm{M}$ website views per year

At $\mathrm{BMC}$, research is always in progress.

Learn more biomedcentral.com/submissions 